\title{
ARTÍCULOS
}

\section{FRAY BARTOLOMÉ DE OLMEDO: UNA RETÓRICA DE LA MERCED EN LA NUEVA ESPAÑA, SIGLOS XVII AL XVIII.}

\author{
Yolanda Guzmán Guzmán \\ El Colegio de Michoacán \\ nehesiyolis@gmail.com
}

Resumen: La presencia de la orden de la Merced en la Nueva España comienza con el paso de fray Bartolomé de Olmedo junto a las huestes de Hernán Cortés como capellán; en la tradición histórica mercedaria, el fraile fue el primer evangelizador del virreinato. El objetivo de este artículo es indagar en cómo, por quiénes y por qué La Merced enfatizó a este capellán del conquistador hasta un siglo después. Se trata de demostrar que, al enfatizar la memoria de Olmedo, los mercedarios novohispanos insertaron a su orden al mismo nivel, o incluso por encima, de las órdenes mendicantes que protagonizaron la cristianización de los naturales. Recurrir a este relato permitió que a lo largo del siglo XVII se construyera un argumento retórico que las autoridades mercedarias utilizaban cada vez que pedían una limosna al rey 0 gestionaban una fundación en las audiencias de México o Nueva Galicia.

Palabras clave: Orden de la Merced, fray Bartolomé de Olmedo, Bernal Díaz del Castillo, Nueva España, conquista, tradición histórica.

Tittle: FRAY BARTOLOME DE OLMEDO: ONE MERCEDARIAN RETHORIC IN NEW SPAIN, $17^{\text {th }}$ TO $18^{\text {th }}$ CENTURY.

Abstract: The presence of the Order of Mercy in New Spain begins with fray Bartolome de Olmedo's steps next to the troops of Hernan Cortes as his Chaplain during the conquest. In the Historic Mercedarian Tradition, this friar was the first missionary in the Viceroyalty, who converted Indians to Christianity. The objective of this article is to inquire into How, Who and Why the Order of Mercy emphasized this Chaplain until the Seventeenth Century. My proposal is to prove that, recovering the forgotten memory of Olmedo allowed Mercedarians to write their role in the history of Evangelization, at the same level, or above, of the Missionary Orders in New Spain. Appealing to this tradition, friars created, or invented, a rhetorical argument, during the Seventeenth, that Mercedarian authorities used every time they asked royal alms or negotiated a new foundation in Audiencias of Mexico and New Galicia.

Keywords: Order of Mercy, fray Bartolomé de Olmedo, Bernal Díaz del Castillo, New Spain, conquest, historic tradition.

Cómo citar este artículo: GUZMÁN GUZMÁN, Yolanda. Fray Bartolomé de Olmedo: una retórica de la Merced en la Nueva España, siglos XVII al XVIII. Naveg@mérica. Revista electrónica editada por la Asociación Española de Americanistas [en línea]. 2020, n. 24. Disponible en: <http://revistas.um.es/navegamerica>. [Consulta: Fecha de consulta]. ISSN 1989-211X. 


\section{Introducción}

En la tradición histórica mercedaria, la presencia de la orden en la Nueva España comienza con los pasos de fray Bartolomé de Olmedo junto a las huestes de Hernán Cortés. Esta tradición empezó a ser contada por los frailes a comienzos del siglo XVII. El objetivo de este artículo es indagar cómo, por quiénes y por qué la Orden de la Merced enfatizó a este capellán del conquistador hasta un siglo después de su vida en Nueva España, a pesar de que ya había fuentes publicadas que hablaban de este mercedario desde la segunda mitad del siglo XVI. Mi propuesta es demostrar que los frailes mercedarios de comienzos del siglo XVII emprendieron la tarea de contar su historia para reafirmar su identidad en la sociedad; al narrar su participación en el descubrimiento y conquista del Nuevo Mundo se dieron cuenta de los privilegios que ameritaba formar parte de la historia de la evangelización para asegurar limosnas o fundaciones en lugares como la Nueva España, en donde la orden carecía de una labor misionera entre los pueblos de indios. Al rescatar del olvido la memoria de Olmedo, los frailes mercedarios novohispanos insertaron a su orden en el mismo nivel, o incluso por encima, de las órdenes mendicantes que protagonizaron la cristianización de los naturales en la Nueva España, como los franciscanos, los dominicos y los agustinos. Recurrir a este relato permitió que a lo largo del siglo XVII se construyera un argumento retórico que las autoridades mercedarias utilizaban cada vez que pedían una limosna regia o una fundación nueva en las audiencias de México o Nueva Galicia. Para afianzar este argumento retórico, la edición y publicación de la obra de Bernal Díaz del Castillo por los mercedarios se convirtió en la fuente impresa de autoridad que los frailes necesitaban.

\section{La formación de una tradición}

La Orden de la Merced, como corporación o institución religiosa y uno de los miembros de la Iglesia católica se ha encargado de escribir y contar su historia por medio de diferentes figuras como los cronistas. Esta es una historia escrita por frailes profesos de la misma orden, quienes se han encargado, especialmente después de 1574, de rescatar documentos que, en algún momento, formaron parte de los archivos de sus conventos; o recabar el testimonio de la memoria de los frailes ancianos y sus contemporáneos; o contar su propia experiencia sobre algún suceso importante en el devenir cotidiano de sus casas. En 1616, el maestro general, fray Francisco de Ribera, institucionalizó el oficio de cronista general de la orden como parte de la conmemoración por los festejos de fundación de la congregación. Además del cronista general, cada convento o provincia comenzó a nombrar a un religioso para que se encargara de dejar testimonio de los principales acontecimientos. Al pensar en estas figuras en el contexto indiano, o del Nuevo Mundo, los testimonios escritos que se han podido rescatar y divulgar son pocos en comparación con los que se conocen para los conventos europeos de La Merced. La compilación de estas obras ha permitido que la Orden de la Merced pueda identificar los principales sucesos y protagonistas de su historia que comienza en 1218 con la comunidad de Pedro Nolasco y que hasta el presente se ha extendido por todo el mundo; el recuento de sus acciones y sujetos forma el corpus de la tradición histórica mercedaria y es la memoria de la orden. 
La tradición histórica mercedaria, es decir, la historiografía sobre la Orden de la Merced escrita por mercedarios puede ser estudiada desde diferentes perspectivas; en esta ocasión me interesan los postulados de autores como Edmundo O'Gorman', John Elliott ${ }^{2}$ y Eric Hobsbawm ${ }^{3}$ en cuanto al análisis de cómo se forma una tradición escrita en el paso del tiempo. Mi interés es aplicar estas propuestas de estudio en la figura de fray Bartolomé de Olmedo para comprender el desarrollo del discurso entorno a este fraile y los usos que hicieron de este sujeto histórico en los siglos XVII y XVIII en Nueva España. Antes de continuar, es pertinente dejar claros algunos conceptos.

Los autores antes citados coinciden en que hay un proceso de invención sobre la forma en que se entienden, en un momento determinado, algunas posturas 0 ideas. Justo este proceso de invención es lo que ha llamado la atención de los historiadores sobre algunos presupuestos en la historia. Edmundo O'Gorman marcó un cambio en la comprensión del hecho histórico sobre el supuesto descubrimiento de América por parte de Cristóbal Colón en 1492 y analiza, por medio del estudio de las obras publicadas y sus autores, la gestación de la percepción de un "descubrimiento" para los europeos, que no contemplaba la postura de los pueblos nativos. De manera similar, Elliott retoma esta idea de indagar en las diferentes percepciones que hubo sobre el encuentro entre el Viejo y el Nuevo Mundo, y centra su atención en el impacto que tuvo la inclusión de América en la dinámica histórica de Europa, más allá de España. Ambos autores, sitúan sus trabajos en el contexto del mundo hispánico de la época moderna. En contraste, Hobsbawm desprende sus postulados desde la realidad del imperio británico del siglo XIX y los nacionalismos europeos posteriores a la invasión napoleónica para la comprensión de las formaciones de identidades en los grupos sociales en la era del capitalismo industrial.

Estos tres autores hablan de una invención porque se trata de posturas, ideas, o tradiciones, que se van creando en el tiempo y en el espacio por sujetos determinados en constante intercambio y negociación con su contexto. Las fuentes que utilizan estos historiadores son muy diversas; en su mayoría son impresos publicados en la época; con los cuales, al colocarlos en una línea temporal, se puede observar el cambio en las percepciones y las añadiduras o extracciones en los relatos. Es justamente este modelo de acercamiento a las fuentes y su análisis en el contexto lo que he tomado como base para este artículo en cuanto al acercamiento a la producción historiográfica mercedaria escrita por mercedarios en los siglos XVII y XVIII.

Opté por esta forma de trabajar porque la invención de una tradición, en este caso, una tradición histórica, tiene como base las crónicas y los textos históricos; los

\footnotetext{
${ }^{1}$ O'GORMAN, Edmundo. La invención de América. Investigación acerca de la estructura histórica del nuevo mundo y del sentido de su devenir. $3^{\text {a }}$ ed. Ciudad de México: Fondo de Cultura Económica, 2006.

${ }_{2}^{2}$ ELLIOTT, John H. El Viejo Mundo y el Nuevo (1492-1650). 3ª ed. Madrid: Alianza Editorial, 2015.

${ }^{3}$ HOBSBAWM, Eric. Introducción: la invención de la tradición. En: HOBSBAWM, Eric y RANGER, Terence (eds.). La invención de la tradición. Barcelona: Editorial Crítica, 2002, pp. 7-21; HOBSBAWM, Eric. La fabricación en serie de tradiciones: Europa, 1870-1914. En: HOBSBAWM, Eric y RANGER, Terence (eds.). La invención de la tradición. Barcelona: Editorial Crítica, 2002, pp. 272-318.
} 
cuales, al ser estudiados y vistos en la línea del tiempo, se observa cómo se fue moldeando la figura de fray Bartolomé de Olmedo, y se logra percibir lo que Hobsbawm señala como las intenciones políticas o sociales asociadas a una tradición. En esta ocasión, me interesa más discutir el proceso de la invención, que el concepto mismo de tradición, porque esta invención es lo que me ayuda a explicar el argumento retórico utilizado por los frailes mercedarios, a ambos lados del Atlántico, para gestionar algún privilegio o limosna regia, así como nuevas fundaciones en la Nueva España.

\section{Los relatos de la Conquista y el capellán de Hernán Cortés}

Los primeros mercedarios que escribieron sobre su presencia en las Indas a finales del siglo XVI y comienzos del XVII recurrieron a las obras de Pedro Mártir de Anglería ${ }^{4}$, Francisco López de Gómara ${ }^{5}$, Antonio de Saavedra Guzmán ${ }^{6}$, Alonso de Ercilla y Zúñiga ${ }^{7}$, Agustín de Zárate y Pedro Cieza de León ${ }^{8}$. Algunos de estos textos hacen mención del fraile Olmedo como capellán de las huestes de Hernán Cortés, pero sin mucho énfasis en su participación directa en la evangelización de los naturales; estos autores estaban más interesados en narrar la historia de la conquista militar, que la espiritual, matiz que van a agregar los escritores mercedarios del siglo XVII.

Los primeros escritos del descubrimiento de un nuevo continente desconocido para los europeos comenzaron a llegar a España desde los viajes de Cristóbal Colón. Conforme fueron ampliándose las expediciones e instalando las instituciones castellanas, las noticias comenzaron a ser más fructíferas. Con respecto a la Nueva España, los cronistas y protagonistas de la conquista del pueblo Mexica no escatimaron su tinta para narrar las batallas, las acciones y las vidas de los principales involucrados.

En 1632 se dio a conocer la primera edición del texto Historia verdadera de la conquista de la Nueva España contada por uno de sus colaboradores: Bernal Díaz del Castillo; la publicación del manuscrito estuvo a cargo de la Orden de la Merced. La obra fue leída y conocida por algunos frailes mercedarios quienes estuvieron interesados en rescatar y darle los honores que merecía a quien reconocieron como el primer mercedario en Nueva España: fray Bartolomé de Olmedo.

Hasta el momento, ha sido imposible afirmar con plena certeza en quién recayó la responsabilidad de hacer la primera edición de la obra de Bernal Díaz del Castillo. Antonio de Solís y Rivadeneyra ${ }^{9}$ atribuyó la edición fray Alonso Remón, quien fue

\footnotetext{
${ }^{4}$ MÁRTIR DE ANGLERÍA, Pedro. Décadas del Nuevo Mundo. Ciudad de México: Editorial Porrúa, 1964.

${ }^{5}$ LÓPEZ DE GÓMARA, Francisco. Crónica de la Nueva España con la conquista de México y otras cosas notables, hechas por el valeroso Hernán Cortés, Marqués del Valle, capitán de su Majestad en aquellas partes. Zaragoza: Casa de Agustín Millán, 1554.

${ }^{6}$ SAAVEDRA GUZMÂN, Antonio de. El peregrino indiano. Edición crítica de María José Rodilla León. Madrid; Frankfurt: Iberoamericana; Vervuert, 2008.

${ }^{7}$ ERCILLA Y ZÚÑNIGA, Alonso de. La Araucana. Madrid: Imprenta Nacional, 1866.

${ }^{8}$ CIEZA DE LEÓN, Pedro. La crónica del Perú. Madrid; Barcelona: Espasa-Calpe, 1932.

${ }^{9}$ SOLÍS Y RIVADENEYRA, Antonio de. Historia de la conquista de Méjico, población y progresos de
} 
nombrado en 1618 cronista general y se le asignó la tarea de escribir una crónica general de la Orden de la Merced, por el aniversario de su fundación; obra que dejó incompleta por su fallecimiento en 1632. Remón había asumido la responsabilidad de empezar a trabajar en la primera edición de la obra de Bernal ${ }^{10}$. Por el momento desconozco a detalle cómo llegó el texto de Bernal a los mercedarios en la Península, y principalmente a manos de Remón.

Quien sucedió a Remón en la tarea de editar la obra de Bernal fue fray Gabriel Ardazo y Santander ${ }^{11}$, pero es una información que no he podido aclarar porque quien fue nombrado sucesor en el cargo de cronista general y con la encomienda de terminar la obra histórica de fray Alonso Remón fue fray Gabriel Téllez, alias Tirso de Molina $^{12}$, por lo que no estoy segura sobre cómo se organizaron los religiosos de La Merced para la edición de la obra de Díaz del Castillo. ${ }^{13}$

El editor mercedario se tomó algunas libertades narrativas que fueron expuestas a partir del siglo XIX y se comenzó a sembrar la incertidumbre sobre los datos de la obra. En 1904, Genaro García publicó el Manuscrito de Guatemala, documento en el que están basadas las ediciones de la obra de Bernal Díaz del siglo XX y con el cual se pudo contrastar la información y anotar las interpolaciones al texto original ${ }^{14}$. Las alteraciones narrativas están relacionadas principalmente con la información sobre fray Bartolomé de Olmedo.

Algunos de los escolios a la obra de Bernal Díaz en la edición mercedaria consisten solo en agregar junto al nombre de fray Bartolomé de Olmedo el calificativo de miembro de la Orden de la Merced o viceversa. Por ejemplo, en la versión del Manuscrito de Guatemala está escrito "nuestro capitán Cortés también tenía calenturas y aun el padre de la Merced"; mientras que en la edición mercedaria se enuncia "nuestro capitán también tenía calenturas, y aun el padre fray Bartolomé de Olmedo, de la Orden de la Merced"15. Las alteraciones que más han llamado la atención de los investigadores han sido aquellas en que se tergiversaron los hechos

la América Septentrional, conocida por el nombre de Nueva España. Edición facsímil. Ciudad de México: Editorial Cosmos, 1997.

${ }^{10}$ LEÓN CÁZARES, María del Carmen. Reforma o extinción. Un siglo de adaptaciones de la orden de Nuestra Señora de la Merced en Nueva España. Ciudad de México: Universidad Nacional Autónoma de México, 2004, pp. 205-210.

${ }^{11}$ Ibídem, p. 211.

12 PENEDO REY, Manuel (O. de M.). Introducción. En: TÉLLEZ, Gabriel (Tirso de Molina). Historia General de la Orden de Nuestra Señora de las Mercedes. T. I. Edición de fray Manuel Penedo Rey. Madrid: Provincia de la Merced de Castilla, 1973, p. XCI.

${ }^{13}$ Por la diferencia cronológica con la vida de Remón, se pensó en alguien más para concluir el trabajo de edición, por ello autores, como María del Carmen León Cázares, atribuyen la edición a fray Gabriel Ardazo y Santander. Como la discusión en torno al editor mercedario de la obra de Bernal sigue vigente, para fines prácticos, he optado por respetar la tradición de atribuir la primera edición al mercedario fray Alonso Remón.

${ }^{14}$ BARBÓN RODRíGUEZ, José Antonio. Prologo. En: DíAZ DEL CASTILLO, Bernal. Historia verdadera de la conquista de la Nueva España (Manuscrito de "Guatemala"). Edición facsímil de José Antonio Barbón Rodríguez. Ciudad de México: El Colegio de México; Universidad Nacional Autónoma de México; Servicio Alemán de Intercambio Académico; Agencia Española de Cooperación Internacional, 2005, pp. I-III.

${ }^{15}$ Ambas citas corresponden al Capítulo LXVI de la obra de Bernal Díaz del Castillo. Las cursivas son mías y las uso para resaltar la interpolación. 
y ocasionaron inconsistencias narrativas en esta primera edición de la obra de Bernal Díaz del Castillo. Las interpolaciones que más me interesan son aquellas en las cuales la figura de Olmedo pasa de ser capellán de huestes a ser misionero:

"[...] y más decía en el cabo de su carta, cómo luego de a poco tiempo que había salido de Méjico Cortés había muerto el buen fray Bartolomé, que era un santo hombre, y que le había llorado todo Méjico, y que le habían enterrado con grande pompa en Señor Santiago, e que los indios habían estado todo el tiempo desde que murió hasta que le enterraron sin comer bocado, e que los padres franciscos habían predicado a sus horas y enterramiento, y que habían dicho dél que era un santo varón, y que le debía mucho el Emperador, pero más los indios; pues si al Emperador le habían dado aquellos vasallos, como Cortés y los demás conquistadores viejos, a los indios les había dado el conocimiento de Dios y ganado sus almas para el cielo; e que había convertido e bautizado más de dos mil quinientos indios en Nueva España, que ansí se lo había dicho el padre fray Bartolomé de Olmedo algunas veces al tal predicador; e que había hecho mucha falta fray Bartolomé de Olmedo, porque con su autoridad e santidad componía las disensiones e ruidos, y hacia bien a los pobres. $Y$ acaba su carta diciendo: 'Esto que aquí escribo a vuestra merced pasa así, y dejélos allá y enviaron[me] preso en una açemila e con grillos aquí estoy' [...]"16.

Esta parte es un episodio de la carta que envía el licenciado Zuazo (Çuaço) a Hernán Cortés informándole sobre lo que había pasado en México desde su partida; el editor mercedario aprovechó el final de la narración del contenido de la carta para agregar información sobre el fallecimiento de fray Bartolomé de Olmedo y sobre sus actividades en la cristianización de los indios de México.

Los arreglos al texto de la edición mercedaria permiten preguntar sobre el interés de los frailes por resaltar la imagen de fray Bartolomé de Olmedo como el primer evangelizador en la Nueva España en la primera mitad del siglo XVII, porque las alteraciones en la narración solo se encuentran en los episodios donde participa Olmedo y no en los relatos bélicos de la conquista ${ }^{17}$. Esto me conduce a preguntar qué estaba pasando en la Orden de la Merced en la Península como para que los mercedarios centraran su atención en resaltar la participación de fray Bartolomé de Olmedo en la conquista como evangelizador y no lo hicieron en la centuria anterior a pesar de que ya se habían publicado otras obras que narraban la participación del mercedario con las huestes de Cortés; y cuál era la finalidad o el objetivo para hacer estas interpolaciones y presentar a un fraile mercedario casi protagonista en las decisiones que tomaba Hernán Cortés.

Las obras publicadas en el siglo XVI y que hacen referencia a fray Bartolomé de Olmedo como capellán de las huestes de Hernán Cortés son:

\footnotetext{
${ }^{16}$ En este fragmento, aparece en letra redondilla lo que aparece en el Manuscrito de Guatemala, y se ha puesto en cursiva lo que fue agregado por el mercedario encargado de la edición de 1632. Ibídem, Cap. CLXXXV, p. 682. Cfr. LEÓN CÁZARES, María del Carmen. Reforma o extinción. Op. cit., pp. 220-221.

${ }^{17}$ Cfr. LEÓN CÁZARES, María del Carmen. Reforma o extinción. Op. cit., pp. 210-223. La autora hace un análisis puntual de las diferentes interpolaciones del texto de Bernal Díaz que resaltan la figura del fray Bartolomé de Olmedo.
} 
- Francisco López de Gómara, Crónica de la Nueva España con la conquista de México y otras cosas notables, hechas por el valeroso Hernán Cortés, Marqués del Valle, capitán de su Majestad en aquellas partes, Casa de Agustín Millán, Zaragoza, 1554.

- Francisco Cervantes de Salazar, Crónica de la Nueva España, 1575.

- Antonio de Herrera y Tordesillas, Historia general de los hechos de los castellanos en las Islas y Tierra Firme del mar Océano, Imprenta Real, Madrid, 1601.

Estas obras mencionan quienes realizan tareas relacionadas con la predicación del Evangelio que suelen ser Hernán Cortés o Jerónimo de Aguilar. La obra que marcó la pauta narrativa para el relato de la conquista de la Nueva España es la obra de López de Gómara.

Para dar una respuesta a las preguntas planteadas anteriormente, propongo que, en la primera mitad del siglo XVII la figura de fray Bartolomé de Olmedo empezó a ser muy importante para la Orden de la Merced por la distinción que ameritaba haber sido los primeros en llegar a cristianizar a los indios en un contexto donde, debido a la relevancia de este proceso histórico, se podían obtener beneficios o privilegios que otorgaba la Corona. Los frailes mercedarios después de la reforma de 1574 recurrieron frecuentemente a argumentar sus peticiones a beneficio de los frailes novohispanos basándose en que fueron los primeros en evangelizar en la Nueva España con las acciones que atribuyeron a fray Bartolomé de Olmedo.

Desde el descubrimiento del Manuscrito de Guatemala, las interpolaciones de la edición mercedaria dejan en claro que el editor de esta obra estaba interesado en llamar la atención sobre fray Bartolomé de Olmedo; además, el editor de la obra se tomó la libertar de agregar datos apócrifos sobre algunos frailes que viajaron con Hernán Cortés cuando volvió a la Nueva España después de gestionar el título de marqués del Valle. Esta información ha sido relatada y analizada por diferentes investigadores y especialista de la obra de Díaz del Castillo.

\section{Primeras noticias de Olmedo en los textos mercedarios escritos desde España, siglo XVI}

Para seguir entendiendo la edición mercedaria de la obra de Bernal Díaz del Castillo, es importante conocer qué sabían los mercedarios sobre fray Bartolomé de Olmedo antes de conocer la versión bernaldina. Las primicias que llegaban a los frailes mercedarios en España, principalmente en la provincia de Castilla, sobre los pasos de su orden por el Nuevo Mundo junto a los descubridores y conquistadores, así como sobre las primigenias fundaciones, fueron agregadas sin muchos detalles en sus crónicas a finales del siglo XVI y las primeras décadas del XVII.

Uno de los pioneros en mencionar los pasos mercedarios en Indias fue fray Felipe de Guimerán. Las noticias sobre la vida de este fraile son escasas. Según Tirso de Molina, él nació en Valencia y pertenecía a una familia noble. Dentro de la orden, él desempeñó los cargos de lector de teología en el convento de Tarragona y 
Tortosa, y fue comendador de los conventos de Játiva, Orihuela, Puche y Valencia. El cargó más importante que ocupó fue el de maestro general de la orden en $1609^{18}$. Como escritor, fray Felipe de Guimerán fue el autor de algunos sermones, epístolas y vidas de santos ${ }^{19}$, así como de constituciones de su orden para las monjas y para los descalzos ${ }^{20}$. Su obra más conocida y difundida tanto entre los mercedarios como fuera de la orden es su obra histórica sobre la virgen de la Merced ${ }^{21}$.

Su obra histórica, llamada Breve historia de la Orden de Nuestra Señora de la Merced, redempcion de cautivos cristianos, y de algunos santos [...], fue publicada en 1591. El autor rescató del olvido algunos nombres de los frailes capellanes que viajaron con las huestes castellanas a la aventura de la conquista:

\begin{abstract}
"Siendo así que frailes de la madre de Dios de la Merced fueron de los primeros descubridores y conquistadores de él [...] dos religiosos nuestros entre otros de los primeros que pasaron a la Nueva España, llamados fray luan [Juan] Zambrano, y fray Marcos Vardon [?], hombres celosísimos, y grandes predicadores, a quien llevaron consigo Hernando Cortés Marqués del Valle, y otros que la fueron a conquistar, haber sido los primeros que con incomparable trabajo, a que los esforzaba el vivo celo de las almas, aprendieron las cuatro lenguas maternas, y naturales de aquellos reinos, y dieron la traza para imprimirse los vocabularios, con que fuese fácil a todos predicar a los Indios, y ponerles la fe en su lengua" ${ }^{\prime 2}$.
\end{abstract}

Es de notar que, al hablar de la conquista de la Nueva España, el autor menciona a los mercedarios que fundaron el convento de Guatemala, y que en ese momento era el enclave más importante para la orden en el virreinato de la Nueva España. Guimerán no hace mención alguna de fray Bartolomé de Olmedo, pero su obra fue leída en el convento mercedario de México, y va a ser un fraile novohispano quien agregue a Olmedo en el relato, antes de la publicación del texto de Bernal Díaz del Castillo y aderece el relato de este cronista. La omisión de Olmedo en la obra de Guimerán se debió a las fuentes que utilizó para las primicias sobre los mercedarios en Indias porque tomó la información de textos peruanos como la obra

\footnotetext{
18 TÉLLEZ, Gabriel (Tirso de Molina). Historia General... Op. cit., t. II, pp. 319-320.

19 Cfr. GUIMERÁN, Felipe (O. de M.). Sermón que predicó el muy reverendo P.M. Fr. Felipe Guimerán, vicario provincial de la Provincia de Aragón de la Orden de Nuestra Señora de la Merced en las fiestas de. B. P. S. Luys Bertrán, Valencia, 1609. GUIMERÁN, Felipe (O. de M.). La insigne y ejemplar vida y muerte del venerable padre y siervo de Dios fray Pedro Nolasco, religioso de la Orden de nuestra señora de la Merced, redención de cautivos [...], impresa en Valencia en casa de luan Chysostomo Garriz junto al molino de Rovella, 1610. GUIMERÁN, Felipe (O. de M.). Epistola Pastoralis, qua subditos invitat ad prefectum suae Regulae Observantiam, 1609.

${ }^{20}$ Cfr. GUIMERÁN, Felipe (O. de M.). Orden judicial para las provincias de América, Valencia, 1609. GUIMERÁN, Felipe (O. de M.). Constituciones reformadas de los Descalzos de la Merced, Impresa en Salamanca, año de 1611. GUIMERÁN, Felipe (O. de M.). Regla y constituciones de las monjas de la Asumpción de nuestra señora de la ciudad de Sevilla de la Orden de Nuestra Señora de la Merced, redención de cautivos con una breve relación de la fundación y de las venerables madres fundadoras del sobredicho monasterio, en Valencia en casa de Pedro Patricio Mey, junto a Saint María, 1614.

${ }^{21}$ Para mayor información sobre las obras de fray Felipe de Guimerán, vid. PLACER LÓPEZ, Gumersindo (O. de M.). Bibliografía mercedaria. T. I. Madrid: Revista Estudios de la Orden de la Merced, 1968, pp. 94-96.

${ }_{22}$ GUIMERÁN, Felipe (O. de M.). Breve historia de la Orden de Nuestra Señora de la Merced, redempcion de cautivos christianos y de algunos santos y personas ilustres della. Valencia: Casa de los Herederos de Juan Navarro, 1591, pp. 58-59.
} 
de Alonso de Ercilla y Agustín de Zárate ${ }^{23}$.

El relato sobre los pasos mercedarios en Indias, dentro de la obra de Guimerán, es apenas una pequeña fracción del texto. Su objetivo central era el relato sobre las devociones a la virgen de La Merced en los diferentes conventos de la orden. Cuando se publica esta obra, la Orden de la Merced estaba comandada por el maestro general, fray Francisco Salazar (1583-1593), quien estaba interesado por aplicar las reformas acordadas en 1574 y por insertar a su congregación en la dinámica que estaban teniendo las demás órdenes de la Península ${ }^{24}$.

El nombre de fray Bartolomé de Olmedo comenzó a aparecer, entre los primeros mercedarios que fueron a las Indias, en la obra de fray Bernardo de Vargas ${ }^{25}$, publicada en 1618 y 1632. Este autor basó su relato sobre la conquista de la Nueva España en Francisco López de Gómara y Pedro Cieza de León. Del primero de estos autores, Vargas rescató la participación de Olmedo como negociador entre Cortés y Pánfilo de Narváez ${ }^{26}$. Párrafos más adelante, Vargas va a relatar que Olmedo convirtió muchos indios al cristianismo e incluso hablaba la lengua mexica, en la cual les predicaba y bautizaba; muy a semejanza, o quizás basado en lo que hacían los frailes de su orden en el Perú ${ }^{7}$.

Asimismo, Vargas dedica unas líneas para aclarar el error en el nombre de Olmedo ${ }^{28}$ que apareció publicado en la obra de Antonio de Saavedra Guzmán:

"Prendiólos Pedro de Yrizio incontinente, y a México a Cortés los ha embiado, para que ellos le informen claramente del distinto y de todo lo pasado. Cortés procedió en esto cuerdamente, que una carta a Narváez ha embiado con fray Pedro de Olmedo, un religioso de buena vida, santo y virtuoso" 29 .

Considero que es posible que Vargas leyera el relato de Guimerán, en donde las lenguas indígenas como herramienta de evangelización eran importantes, y lo añadiera al episodio de Olmedo. Además de fray Bartolomé, también menciona a fray Juan de Zambrana como acompañante de Hernán Cortés y a fray Juan [?] Vardón [?] $]^{30}$.

\footnotetext{
${ }^{23}$ ZÁRATE, Agustín de. Historia del descubrimiento y conquista del Perú. Edición de Franklin Pease G. Y. y Teodoro Hampe Martínez. Lima: Pontificia Universidad del Perú; Fondo editorial, 1995.

${ }^{24}$ TAYLOR, Bruce. Structures of Reform. The Mercedarian Order in the Spanish Golden Age. Leiden; Boston; Köln: Editorial Brill, 2000.

${ }_{25}$ VARGAS, Bernardo de (O. de M.). Chronica sacri et militaris Ordinis B. Mariae de Mercede Redemptionis captivorvm. Palermo: Ionanes Baptista Maringus, 1618, t. I; 1632, t. II.

${ }^{26}$ Ibídem, t. I, Cap. XXV, p. 383.

27 "Fratrem Bartholomaeum de Olmedo ex Prouincia Castellae, quem similiter suum specialem Consiliarium deputavit. Preuenit igitur Dominus Ferdinandus Cortes ad Mexicanum Regnum, seu nouam Hispaniam anno 1518. [...] Vnde Christiani caeperunt Ecclesiale aedificare, \& Sacerdotes verbum Dei seminare. Presertim magister Olmedo, qui linguam Mexicanam cum suffucuenter sciret; pro rostris concionabatur, \& plures Indos a se conuersos baptizauit”; Ibídem, t. I, Cap. XXV, p. 383.

28 "(licet in nomine errorem commiserit, cum Petro nominando)"; Ibídem, t. I, Cap. XXV, p. 385.

${ }^{29}$ SAAVEDRA GUZMÁN, Antonio de. El peregrino indiano. Op. cit., Canto Doce, p. 236.

${ }^{30}$ Con este último tengo duda si se trata del mismo sujeto de quien habla Guimerán, o de otro fraile que coincide en el apellido. En el texto en latín de Vargas los nombres son "loanes Zambrana" y "loanes de Vardon"; VARGAS, Bernardo de (O. de M.). Chronica sacri et militaris Ordinis B. Op. cit., t. I, Cap. XXV, pp. 382-385.
} 
Hasta este momento, el nombre de fray Bartolomé de Olmedo fue tomado de los relatos que circulaban sobre la conquista de la Nueva España. En las décadas de 1610 y 1620, entre los mercedarios guatemaltecos comenzó a hacer ruido la versión de la conquista contada por Bernal Díaz del Castillo. Antes de su impresión, los religiosos de La Merced en Guatemala mantenían contacto con los descendientes del autor, de quien obtuvieron el manuscrito para su publicación, y además se encargaron de refrendarlo como auténtico. El provincial fray Francisco Ximénez mandó certificar el contenido del manuscrito de Bernal Díaz del Castillo que poseía don Juan Luis del Castillo y Cárcamo, regidor de Guatemala, e hijo del autor; el escribano de la audiencia dio por certera la información contenida sobre fray Bartolomé de Olmedo oficiando misa y poniendo la cruz en varios lugares durante sus andanzas con Hernán Cortés ${ }^{31}$. Esta movilización de los frailes guatemaltecos es el eslabón que ayuda a comprender, tanto la edición mercedaria de la obra de Bernal Díaz del Castillo, como la circulación de este texto entre los frailes a ambos lados del Atlántico.

Sin duda, desde España, el mercedario que más puso énfasis en las acciones de fray Bartolomé de Olmedo fue fray Alonso Remón ${ }^{32}$, quien además participó en la edición del texto de Bernal. Él nació en Valencia, quizá en el año 1560, y murió en 1632; al igual que los otros cronistas, sus datos biográficos también son parcos. Profesó en la Orden de la Merced en 1605, y debido a su fama como escritor de comedias, el maestro general, fray Francisco de Ribera, lo nombró cronista general de la orden y le pidió que escribiera una historia con el motivo de la conmemoración de los 400 años de fundación de la comunidad de Pedro Nolasco; antes de este momento, este cargo no existía con esta solemnidad dentro de la comunidad mercedaria $^{33}$.

Como literato, fray Alonso Remón mantuvo contacto con otros dramaturgos de su época, como Lope de Vega y Miguel de Cervantes. En su obra La casa de la Razón y el desengaño, publicada en 1625, Remón hace un listado de su producción literaria hasta ese momento ${ }^{34}$. Sus textos fueron aumentando en cantidad en los años siguientes, porque continuó escribiendo y publicando comedias y vidas de santos; y al igual que otros mercedarios, durante las celebraciones por el reconocimiento del culto inmemorial a su fundador, escribió sobre las fiestas en honor a Pedro Nolasco ${ }^{35}$.

El texto de historia de fray Alonso Remón ${ }^{36}$ está dividido en dos tomos, el

\footnotetext{
${ }^{31}$ Archivo General de Indias (en adelante AGI), Guatemala 176. Guatemala, 19 de abril de 1619, ff. 25r.-26v.

32 REMÓN, Alonso (O. de M.). Historia General de la Orden de Nuestra Señora de la Merced, Redención de Cautivos. T. II. Madrid: Luis Sánchez, impresor del Rey Nuestro Señor, 1633.

${ }^{33}$ ZURIAGA SENENT, Vicent Francesc. La imagen devocional en la Orden de la Merced. Tradición, formación, continuidad y variantes. Valencia: Institució Alfons El Magnànim, 2007, p. 129.

${ }^{34}$ Algunas de las obras citadas en su texto son: De la concepción purísima de N. Sra. [...], Madrid, 1616. Historia de la imagen de la Madre de Dios de los Remedios que está en el convento de N. Señora de la Merced de Madrid [...], Madrid, 1617. Vida y muerte misteriosa del gran siervo de Dios Gregorio López, natural de Madrid [...], Madrid, 1617.

${ }^{35}$ Para mayor información de las obras de fray Alonso Remón, vid. PLACER LÓPEZ, Gumersindo (O. de M.). Bibliografía mercedaria. Op. cit., t. II, pp. 586-604.

${ }^{36}$ REMÓN, Alonso (O. de M.). Historia General de la Orden... Op. cit., 1618, t. I; 1633, t. II.
} 
primero de ellos fue publicado el mismo año que la crónica de Vargas: 1618; pero el segundo tomo fue impreso en 1633, después de su muerte; es en este, donde se narran las acciones de la orden en el Nuevo Mundo. En la historia de Remón, las loas para Olmedo continúan; se enfatiza la idea de ser el primero en evangelizar en la Nueva España; y con ello, La Merced como la primera orden en misionar. Al juntar la tradición histórica que se había gestado con Vargas y sustentarla con la obra de Bernal Díaz del Castillo, el texto de Remón aglutina el argumento de fray Bartolomé de Olmedo como evangelizador de la Nueva España y el primero en hacer esta labor:

"[...] de modo que la primera Missa que se dijo en la Nueva España; y Tierra Firme, y la primera Cruz que se puso, y primera Imagen de Nuestra Señora, y el primero sermón que se predicó, y la primera persona que se bautizó y la primera obediencia que se dio a la Magestad Católica y Cesarea del Emperador Carlos Quinto [...] todo se hizo; manejó, y obró por mano del Religioso de la Merced fray Bartolomé de Olmedo"37.

A partir de esto, los autores mercedarios de las provincias españolas van a recurrir como primera fuente a las versiones de estos dos autores, Vargas y Remón, más la obra de Bernal. A la figura de fray Bartolomé de Olmedo la van a ir adornando con más detalles, como el número de indios que convirtió, la variedad de lenguas que habló, e incluso los milagros relacionados con la conversión de los indios que llegó a presenciar durante la conquista. Un ejemplo de esto es la obra de Tirso de Molina; debo señalar que este texto no se publicó en el momento en que se escribió (1639), sino hasta 1973, pero es una muestra de cómo se fue engranando la figura de Olmedo entre los autores que escribían desde España:

\begin{abstract}
"Después, quando en el año mil quinientos y veinte, entró Cortés en aquellas dilatadíssimas provincias, que ennobleció con el apellido de la Nueva España, acompañándole quatrocientos españoles, solo fray Bartolomé de Olmedo, de nuestro hábito, y un clérigo presbítero, llamado Juan Díaz, fueron, el nuestro por capellán mayor y el otro por su teniente [...] La primera iglesia que adquirió la fe católica en México, no tuvo por cabeza ni artífice sino a fray Bartolomé de Olmedo [...] herido de muerte por sus vasallos mismos Moctezuma, le bautizó nuestro solícito fray Bartolomé de Olmedo y murió aquel gran monarca conociendo la verdad de nuestra religión sagrada" ${ }^{38}$.
\end{abstract}

La versión de fray Gabriel Téllez está basada en el relato contado por fray Alonso Remón y la edición mercedaria de la obra de Bernal Díaz del Castillo, en las cuales, el principal protagonista del trabajo de cristianización en la Nueva España, junto a Hernán Cortés, fue fray Bartolomé de Olmedo.

Para los mercedarios españoles el relato de Olmedo forma parte de la tradición histórica de su orden y queda en el contexto de la historia general de La Merced como un episodio más; en cambio, como veremos a continuación, los frailes novohispanos, empezando por los guatemaltecos, hicieron uso del relato de Olmedo para justificar que fue el primer misionero de la Nueva España; y fortalecieron al

\footnotetext{
${ }^{37}$ REMÓN, Alonso (O. de M.). Historia General de la Orden... Op. cit., 1618, t. II, p. 105.

38 TÉLLEZ, Gabriel (Tirso de Molina). Historia General... Op. cit., t. I, pp. 446-447.
} 
argumento con la idea de haber sido los primeros en convertir a los naturales al cristianismo en la Nueva España, antes que las demás órdenes misioneras.

\section{Fray Bartolomé de Olmedo y los mercedarios novohispano, siglos XVII y XVIII}

El primero en poner el nombre de fray Bartolomé de Olmedo en una obra y publicarlo fue el mercedario fray Luis de Cisneros ${ }^{39}$, quien fue uno de los primeros criollos en profesar en el convento de la ciudad de México y quien en 1616 escribió un relato sobre las procesiones de la virgen de Los Remedios a esta urbe. En este texto dedicó unas cuantas frases para relatar la participación de Olmedo en la conquista junto con los otros frailes que mencionó Guimerán. El objetivo de esta obra no era la historia de La Merced en el virreinato, quizás porque el convento mercedario en la Ciudad de México tenía poco más de 10 años de haber sido fundado, y en la memoria de la sociedad, las noticias sobre La Merced se relacionaban con sus actividades en la Audiencia de los Confines:

"Y la primera Misa que se dijo fue de fraile de mi Orden, porque el Marqués del Valle trajo, cuando vino a la conquista, de la Isla de Santo Domingo con licencia de su Prelado a fray Bartolomé de Olmedo, fray Juan de Zamorano, fray Marcos Bardón [?], que se hallaron en toda ella y decían Misa a todo el ejército, confesaban y predicaban, catequizaron y bautizaron infinitos indios y se fueron convirtiendo con Pedro de Alvarado, y los demás conquistadores que pasaron a Guatemala, donde estuvieron muchos años haciendo este oficio. Y corrían desde Nicaragua hasta los confines de Chiapa, sin parar, [...] Ellos fueron los que aprendieron las cuatro lenguas maternas, imprimieron vocabularios e hicieron instrucciones y catecismos en estas lenguas, con que se facilitó la doctrina" ${ }^{\prime 4}$.

Este fragmento de Cisneros es interesante porque para los frailes del virreinato novohispano era claro que su historia comenzaba con estos tres nombres, y el brinco cronológico entre la participación de Olmedo en la conquista (1519-1524), y la fundación del convento en la ciudad de México en 1594 quedaba subsanado por el desempeño de los religiosos en el reino de Guatemala; para los frailes de la primera mitad del siglo XVII, no era relevante preguntarse por qué no hubo mercedarios que continuaran la labor de este fraile en la Audiencia de México.

Es de notar que la obra de Cisneros fue publicada antes que las obras de Díaz del Castillo y Remón. El autor era conocido entre los frailes guatemaltecos porque en 1619, el padre Ambrosio del Castillo, presbítero y rector del seminario de Nuestra Señora de la Asunción de Guatemala, como uno de los testigos para una información de partes realizada por el provincial mercedario, fray Francisco de Ximénez, dio testimonio de la participación de Cisneros en la Universidad de México como catedrático ${ }^{41}$, cargo que ocupó en $1617^{42}$. Esto demuestra la circulación de las

${ }^{39}$ CISNEROS, Luis de (O. de M.). Historia del principio, origen, progresos y venidas a México y milagros de la santa imagen de Nuestra Señora de los Remedios extramuros de México. Edición facsímil de Francisco Miranda. Zamora, México: El Colegio de Michoacán, 1999.

${ }_{40}$ Ibídem, p. 41.

${ }^{41}$ AGI, Guatemala 176. Guatemala, 9 de abril de 1619, f. $19 \mathrm{v}$.

${ }^{42}$ CISNEROS, Luis de (O. de M.). Historia del principio... Op. cit., pp. 2-3. 
noticias entre los frailes mercedarios, por lo que es posible que las noticias sobre Olmedo hayan sido tomadas por la cercanía que tenían los religiosos con los descendientes de Díaz del Castillo, más los datos publicados en los primeros textos sobre la Conquista, como López de Gómara.

En 1688, el cronista mercedario, fray Francisco de Pareja ${ }^{43}$ terminó de escribir su obra y dedicó el primer estado de su libro a la defensa de fray Bartolomé de Olmedo como el primer evangelizador y misionero de la Nueva España entre los naturales, casi olvidando su faceta de capellán. Él nació en la Nueva Galicia, en el poblado de Los Ramos; sus padres eran también nacidos en el virreinato novohispano, pero sus abuelos eran oriundos de España ${ }^{44}$; su abuelo paterno, don Francisco Pareja, fue uno de los primeros oidores de la Nueva Galicia. Él formó parte de los primeros criollos en ingresar a La Merced en la Ciudad de México en la primera mitad del siglo XVII; se destacó por haber ocupado la cátedra de vísperas de teología y por haber sido rector del Colegio de Comendadores de San Ramón Nonato ${ }^{45}$.

En esta obra, el autor compiló la tradición histórica que se había gestado hasta ese momento, y basó la mayor parte de sus argumentos apologéticos en Bernal Díaz de Castillo. La necesidad de la defensa de esta figura mercedaria surgió por la publicación de algunas crónicas escritas por otros religiosos fuera de su orden, quienes, según Pareja, no le daban el suficiente crédito o mérito a Olmedo; por ello impugnó a varios de estos textos:

"Han quedado ya desvanecidas las calumnias con que algunos han procurado desdorar la gloria de haber sido religiosos de mi sagrada religión los primeros que enarbolaron el estandarte de nuestra fe católica en este reino; y para más prueba de esta verdad, se irán individuando los hechos de Fr. Bartolomé de Olmedo en la prosecución de su ministerio apostólico a que con tan fervoroso espíritu se dedicó, ofreciéndose en sacrificio a Dios, para perder la vida si fuese necesario en servicio de ambas Magestades"46.

El objetivo de la obra es contar la historia de la provincia mercedaria de La Visitación, y quiero agregar que es la primera crónica mercedaria sobre esta provincia novohispana. Para Pareja, los conventos mercedarios de esta provincia eran los herederos directos de las acciones de Olmedo y en él sustentaban su existencia; es decir, los pasos de Olmedo los siguieron los mercedarios que fundaron el convento de la Ciudad de México. Esta obra tiene la particularidad de que los protagonistas de los relatos son los mercedarios de origen criollo, como el autor; este matiz le permite a Pareja enraizar la historia de La Merced con la historia

\footnotetext{
${ }^{43}$ PAREJA, Francisco (O. de M.). Crónica de la Provincia de la Visitación de Nuestra Señora de la Merced, Redención de Cautivos de la Nueva España. Edición facsímil. San Luis Potosí: Archivo Histórico del Estado de San Luis Potosí, 1989.

${ }^{44}$ FERNÁNDEZ DE RECAS, Guillermo. S. Aspirantes americanos a cargos del Santo Oficio. Sus genealogías ascendentes Edición de Manuel Romero de Terreros. Ciudad de México: Librería de Manuel Porrúa, 1956, p. 110.

45 BERISTAIN DE SOUZA, José Mariano. Biblioteca hispano americana septentrional o catálogo y noticias de los literatos. 1521-1825. T. IV. Ciudad de México: Fuente Cultural, 1883, p. 105.

${ }^{46}$ PAREJA, Francisco (O. de M.). Crónica de la Provincia... Op. cit., t. I, p. 21.
} 
de la Nueva España desde la conquista, y el eslabón es fray Bartolomé de Olmedo.

La publicación de este texto no se hizo sino hasta 1882, y tan solo el tomo I. La obra completa fue publicada en 1989. Sin embargo, esto no impidió que el manuscrito circulara entre los frailes mercedarios del convento de México.

En el siglo XVIII, en la década de 1770 , fray Cristóbal de Aldana ${ }^{47}$ fue asignado con la tarea de escribir la historia de la provincia de La Visitación y para ella retomó algunos episodios de la obra de Pareja. Desafortunadamente, Aldana no alcanzó a completar su encomienda porque falleció. Este texto incompleto fue publicado en 1953 y por el momento desconozco su circulación entre los frailes mercedarios, porque la obra más citada es la de su antecesor. Sin el matiz apologético de Pareja, Aldana menciona que:

"El P. F. Bartolomé se dedicó desde luego al consuelo de los Indios, y a su instrucción; defendiéndoles de las vejaciones de los Españoles, asistíalos en sus enfermedades, y los socorría en sus miserias. Instruía a los Niños, para ganar a sus padres: movía, y convencía a los Christianos, para que edificasen a los Idólatras, y desta suerte valiéndose de los unos para la conversión de los otros, procuraba extender por todas partes el buen orden, y Fe de JesuCrhisto"48.

La particularidad de la obra de Aldana fue su intento por ser más crítico ante el trabajo de su predecesor en algunos episodios de la historia de la provincia mercedaria; sin embargo, el modelo establecido por Pareja de iniciar las andanzas mercedarias con fray Bartolomé de Olmedo como misionero no cambió. En esta obra, las acciones de fray Bartolomé de Olmedo no distan mucho de lo que las crónicas de las provincias de otros religiosos relatan sobre los primeros frailes que evangelizaron en la Nueva España.

\section{La retórica de La Merced en la gestión de privilegios, limosnas y fundaciones, siglos XVI y XVII}

El argumento retórico de los frailes mercedarios en Nueva España consistió en afirmar que fueron los primeros y más antiguos religiosos en predicar el evangelio y convertir a los naturales, desde la conquista de la Nueva España, junto al marqués del Valle, y fue una labor protagonizada por fray Bartolomé de Olmedo, quien cristianizó a los indios y habló al menos una de las lenguas indígenas. Esto permitió que las autoridades novohispanas de los diferentes conventos hicieran peticiones al rey basándose en este argumento.

Desde Guatemala, en las últimas décadas del siglo XVI, y las primeras del siglo $X V I I$, los frailes mercedarios realizaron varias informaciones de oficio ante la Audiencia de los Confines, o de Guatemala, con diferentes propósitos. En su mayoría coinciden en pedir alguna limosna, como la del vino y aceite, para sus fundaciones en los pueblos de indios, debido a la pobreza de sus doctrinas y a sus

\footnotetext{
${ }^{47}$ ALDANA, Cristóbal de (O. de M.). Crónica de la Merced de México. Edición facsímil de Jorge Gurría Lacroix. Ciudad de México: Universidad Nacional Autónoma de México; Biblioteca Nacional de México, 1953.

${ }^{48}$ Ibídem, p. 15.
} 
limitaciones para sustentar a los frailes; o pedir dádivas para arreglar desperfectos en los conventos ocasionados por los terremotos. En estas informaciones de oficio, los testigos debían responder varias preguntas sobre la participación de los mercedarios en la evangelización de los naturales para confirmar que la orden había sido la primera en llegar al reino de Guatemala, en fundar un convento y en cristianizar a los naturales.

De las diversas informaciones que hicieron los frailes guatemaltecos, me interesa rescatar la hecha en 1619 porque refleja lo que he mencionado sobre Olmedo. En abril de este año, los mercedarios presentaron una información de oficio para probar que ellos fueron los primeros religiosos que llegaron a la ciudad de Guatemala y fundaron un convento que no se había despoblado hasta esa fecha, en el cual impartían cursos para formar a sus frailes y a algunos hijos de los primeros pobladores. Uno de los objetivos de esta información era demostrar que la versión de Bernal Díaz del Castillo no era errónea al mencionar las acciones de fray Bartolomé de Olmedo. Todos los testigos presentados confirmaron la información solicitada por los frailes, y basaban su conocimiento en los relatos de conquistadores y primeros pobladores del reino de Guatemala, de los ocho testigos, la mitad de ellos sustentaba su información en el relato de Bernal:

\begin{tabular}{|c|c|}
\hline \multicolumn{2}{|c|}{ Testigos } \\
\hline Nombre & Oficio \\
\hline Sancho de Barahona & Encomendero \\
\hline Don García Castellanos *49 & Encomendero \\
\hline Nuño Saez [?] Marroquín * & Encomendero \\
\hline Pedro del Castillo Becerra * & Conquistador y juez oficial \\
\hline Francisco de Abrejo [?] & Vecino de Guatemala \\
\hline Lucas Deardón [?] & Encomendero \\
\hline Padre Ambrosio del Castillo * & Presbítero y rector del seminario \\
\hline Juan Gutiérrez de Xivaxa [?] & Encomendero \\
\hline
\end{tabular}

Fig. 1: Testidos. Fuente: AGI, Guatemala 176. Guatemala, 9 de abril de 1619. Elaboración de la autora.

Uno de los testigos presentados era el padre Ambrosio del Castillo, sobre quien he hecho mención anteriormente y quien, al contestar la pregunta sobre la antigüedad del convento mercedario ${ }^{50}$, respondió elocuentemente citando el texto de Bernal Díaz del Castillo, su abuelo, en donde se narraban las andanzas de fray Bartolomé de Olmedo:

\footnotetext{
${ }^{49}$ Los nombres marcados con * son los testigos que sustentaron sus respuestas en la versión contada por Bernal Díaz del Castillo.

50 "Si saben, han visto o oído decir que el dicho convento de nuestra señora de las mercedes es el más antiguo y el que primero se fundó en esta ciudad al tiempo de la conquista, pacificación y reducción y población de esta tierra y que los religiosos de ella fundaron y establecieron [incompleto] a dicha orden ayudaron con su predicación, virtud y buen ejemplo, vida regular y administración de los sacramentos a la conquista pacificación y reducción de los naturales a nuestra santa fe católica de que han resultado y resultan tantos y tan conocidos frutos en servicio de ambas majestades divina y humana y aumento de nuestra santa fe y religión cristiana dilatación y extensión de la Iglesia". AGI, Guatemala 176. Guatemala, 9 de abril de 1619, f. 1 v.
} 
"[...] el primer sacerdote que celebró el santo sacrificio de la misa y predicó el santo evangelio y bautizó cuando el marqués del valle vino a este nuevo mundo de las indias fue un religioso de nuestra señora de la merced nombrado fray Bartolomé de Olmedo hombre de mucha religión y santidad de letras y que ayudó mucho al marqués del valle en los negocios de la pacificación de estas partes y Nueva España lo cual sabe este testigo por haberlo leído en una historia original que tiene en su poder cuyo autor es Bernal Díaz del Castillo su abuelo uno de los primeros descubridores y conquistadores de las Indias los cuales son papeles" ${ }^{21}$.

Con esta información de oficio, los frailes mercedarios tenían un documento que avalaba su antigüedad y sus méritos en la evangelización. Este escrito ayuda a entender el interés de los mercedarios por publicar la obra de Bernal Díaz del Castillo y las interpolaciones de su editor.

En el siglo XVII, los frailes del convento mexicano compartieron espacio con las órdenes misioneras de la Nueva España (franciscanos, dominicos, agustinos y jesuitas), pero ellos no pudieron administrar pueblos de indios porque la licencia que les habían otorgado para la fundación del convento en 1594 se los prohibía. Sin embargo, esto no fue impedimento para que los religiosos apelaran a la figura de Olmedo y le recordaran a las autoridades regias y a las demás órdenes religiosas que ellos fueron los primeros en misionar.

Un ejemplo de esto fue fray Francisco de Ribera, quien conoció a los frailes del virreinato novohispano en los primeros años del siglo XVII cuando fue nombrado vicario general (1606-1610) para estos conventos. Como maestro general (16161618) de la Orden de La Merced, Ribera se encargó de la gestión de más fundaciones y la formación de la provincia de La Visitación de la Nueva España. Para la acreditación de la provincia, eran necesarios más conventos, para ello, Ribera, basándose en su experiencia como visitador, y aprovechando su cargo de gestión como maestro general, escribió al Consejo de Indias para pedir la fundación de nuevos conventos de su orden y conseguir la separación administrativa de los conventos fundados en la Audiencia de México, de las casas mercedarias en la Audiencia de Guatemala; uno de los argumentos, además de las distancias, fue la labor de evangelización durante la conquista de la Nueva España ${ }^{52}$.

Cuando fue obispo de Guadalajara (1618-1629), fray Francisco de Ribera gestionó un convento de su orden para esta ciudad, y cuando ocupó el mismo oficio en Michoacán (1630-1637) apoyó a los frailes de su orden en Valladolid. Apelando a la antigüedad de su orden y al trabajo de fray Bartolomé de Olmedo, consiguió que el cabildo eclesiástico de Michoacán incluyera en las fiestas de guardar y con solemnidad la celebración de la fiesta de San Pedro Nolasco, fundador de La Merced, y se agregara una imagen en la iglesia catedral de la capital episcopal:

"[...] devoción del glorioso santo, estar determinado por el concilio mexicano que todos los gloriosos patriarcas de las religiones fuesen días festivos de guardar atendiendo a que los hijos de las dichas religiones habían acudido, fomentado las conversiones de los infieles en estos reinos y siendo así que los religiosos de

\footnotetext{
51 Ibídem, ff. 18r.-19r.

52 AGI, Guatemala 175. Madrid, 1616.
} 
nuestra señora de la Merced habían sido los primeros que pasaron a los reinos a la dicha conversión",53.

En el siglo XVII, en la Nueva España, la Orden de la Merced continuó creciendo en conventos y prosiguió apelando a la memoria de fray Bartolomé de Olmedo como primer evangelizador. En tiempos recientes, esta tradición es rememorada y actualizada, en cuanto a que los frailes siguen estudiando a la figura de Olmedo y sus acciones en la conversión de naturales. Los trabajos hechos en el siglo XX preservan la esencia del argumento sobre la participación de Olmedo en las andanzas junto a Hernán Cortés y sus aportes a la predicación del evangelio entre los naturales ${ }^{54}$.

\section{Consideraciones finales}

La construcción de una tradición se da a lo largo del tiempo y sirve para gestar una identidad y cohesión en un grupo social. A lo largo del siglo XVII, la Orden de la Merced, por medio de sus cronistas, se encargó de ir formando una tradición en torno a las acciones y protagonismos de fray Bartolomé de Olmedo en la conquista de la Nueva España junto a Hernán Cortes. Esta tradición histórica forma parte de la identidad de la orden hasta el presente, y les recuerda a sus miembros su participación en un momento importante de la historia del mundo. Fray Bartolomé de Olmedo sigue siendo un referente para La Merced de sus pasos por el Nuevo Mundo y de sus aportaciones en la conversión de los naturales.

El argumento retórico elaborado por los mercedarios del siglo XVII y utilizado por sus frailes novohispanos les permitió a estos últimos tener una tradición histórica legitimadora de su presencia en las Audiencias de México y Nueva Galicia. La retórica mercedaria novohispana favoreció que los religiosos buscaran un lugar entre las órdenes religiosas misioneras, quienes a principios del siglo XVII se estaban enfrentando la amenaza de la secularización de sus doctrinas y escribían con nostalgia las glorias de sus acciones en la centuria anterior. Con esta retórica mercedaria, sus frailes novohispanos justificaron su presencia y pudieron ampliar el número de conventos que formaban la provincia de La Visitación.

Asimismo, con este argumento retórico, los frailes mercedarios se incluyeron en la dinámica de las órdenes misioneras (franciscanos, dominicos, agustinos y jesuitas) quienes también estaban contando sus orígenes para legitimar su permanencia en los pueblos de indios que doctrinaban. Debido a que La Merced no administró pueblos de indios, su retórica legitimaba su establecimiento en las principales ciudades capitales de los obispados, o en las villas de españoles donde se establecieron con ayuda de las familias locales.

\footnotetext{
53 Archivo Capitular del Cabildo Catedral de Morelia (en adelante ACCCM), Libro 3, Exp. 574. Valladolid, 17 de enero de 1634, ff. 300v.-301r.

54 PLACER LÓPEZ, Gumersindo (O. de M.). Fray Bartolomé de Olmedo. Capellán de los conquistadores de México. Madrid: Artes Gráficas H. de la Guardia Civil, 1961; CASTRO SEOANE, José. El P. Fray Bartolomé de Olmedo, capellán de Cortés. Ciudad de México: Editorial Jus, 1958.
} 


\section{Bibliografía}

ALDANA, Cristóbal de (O. de M.). Crónica de la Merced de México. Edición facsímil de Jorge Gurría Lacroix. Ciudad de México: Universidad Nacional Autónoma de México; Biblioteca Nacional de México, 1953.

BERISTAIN DE SOUZA, José Mariano. Biblioteca hispano americana septentrional o catálogo y noticias de los literatos. 1521-1825. T. IV. Ciudad de México: Fuente Cultural, 1883.

CASTRO SEOANE, José. El P. Fray Bartolomé de Olmedo, capellán de Cortés. Ciudad de México: Editorial Jus, 1958.

CIEZA DE LEÓN, Pedro. La crónica del Perú. Madrid; Barcelona: Espasa-Calpe, 1932.

CISNEROS, Luis de (O. de M.). Historia del principio, origen, progresos y venidas a México y milagros de la santa imagen de Nuestra Señora de los Remedios extramuros de México. Edición facsímil de Francisco Miranda. Zamora, México: El Colegio de Michoacán, 1999.

DÍAZ DEL CASTILLO, Bernal. Historia verdadera de la conquista de la Nueva España (Manuscrito de "Guatemala"). Edición facsímil de José Antonio Barbón Rodríguez. Ciudad de México: El Colegio de México; Universidad Nacional Autónoma de México; Servicio Alemán de Intercambio Académico; Agencia Española de Cooperación Internacional, 2005.

ELLIOTT, John H. El Viejo Mundo y el Nuevo (1492-1650). 3ª ed. Madrid: Alianza Editorial, 2015.

ERCILLA Y ZÚÑIGA, Alonso de. La Araucana. Madrid: Imprenta Nacional, 1866.

FERNÁNDEZ DE RECAS, Guillermo. S. Aspirantes americanos a cargos del Santo Oficio. Sus genealogías ascendentes Edición de Manuel Romero de Terreros. Ciudad de México: Librería de Manuel Porrúa, 1956.

GUIMERÁN, Felipe (O. de M.). Breve historia de la Orden de Nuestra Señora de la Merced, redempcion de cautivos christianos y de algunos santos y personas ilustres della. Valencia: Casa de los Herederos de Juan Navarro, 1591.

HOBSBAWM, Eric. Introducción: la invención de la tradición. En: HOBSBAWM, Eric y RANGER, Terence (eds.). La invención de la tradición. Barcelona: Editorial Crítica, 2002, pp. 7-21.

HOBSBAWM, Eric. La fabricación en serie de tradiciones: Europa, 1870-1914. En: HOBSBAWM, Eric y RANGER, Terence (eds.). La invención de la tradición. Barcelona: Editorial Crítica, 2002, pp. 272-318. 
LEÓN CÁZARES, María del Carmen. Reforma o extinción. Un siglo de adaptaciones de la orden de Nuestra Señora de la Merced en Nueva España. Ciudad de México: Universidad Nacional Autónoma de México, 2004.

LÓPEZ DE GÓMARA, Francisco. Crónica de la Nueva España con la conquista de México y otras cosas notables, hechas por el valeroso Hernán Cortés, Marqués del Valle, capitán de su Majestad en aquellas partes. Zaragoza: Casa de Agustín Millán, 1554.

MÁRTIR DE ANGLERÍA, Pedro. Décadas del Nuevo Mundo. Ciudad de México: Editorial Porrúa, 1964.

O'GORMAN, Edmundo. La invención de América. Investigación acerca de la estructura histórica del nuevo mundo y del sentido de su devenir. $3^{\underline{a}}$ ed. Ciudad de México: Fondo de Cultura Económica, 2006.

PAREJA, Francisco (O. de M.). Crónica de la Provincia de la Visitación de Nuestra Señora de la Merced, Redención de Cautivos de la Nueva España. Edición facsímil. San Luis Potosí: Archivo Histórico del Estado de San Luis Potosí, 1989.

PLACER LÓPEZ, Gumersindo (O. de M.). Fray Bartolomé de Olmedo. Capellán de los conquistadores de México. Madrid: Artes Gráficas H. de la Guardia Civil, 1961.

PLACER LÓPEZ, Gumersindo (O. de M.). Bibliografía mercedaria. Madrid: Revista Estudios de la Orden de la Merced, 1968.

REMÓN, Alonso (O. de M.). Historia General de la Orden de Nuestra Señora de la Merced, Redención de Cautivos. Madrid: Luis Sánchez, impresor del Rey Nuestro Señor, 1618, t. I; 1633, t. II.

SAAVEDRA GUZMÁN, Antonio de. El peregrino indiano. Edición crítica de María José Rodilla León. Madrid; Frankfurt: Iberoamericana; Vervuert, 2008.

SOLÍS Y RIVADENEYRA, Antonio de. Historia de la conquista de Méjico, población y progresos de la América Septentrional, conocida por el nombre de Nueva España. Edición facsímil. Ciudad de México: Editorial Cosmos, 1997.

TAYLOR, Bruce. Structures of Reform. The Mercedarian Order in the Spanish Golden Age. Leiden; Boston; Köln: Editorial Brill, 2000.

TÉLLEZ, Gabriel (Tirso de Molina). Historia General de la Orden de Nuestra Señora de las Mercedes. Edición de fray Manuel Penedo Rey. Madrid: Provincia de la Merced de Castilla, 1973.

VARGAS, Bernardo de (O. de M.). Chronica sacri et militaris Ordinis B. Mariae de Mercede Redemptionis captivorvm. Palermo: Ionanes Baptista Maringus, 1618, t. I; 1632, t. II. 
ZÁRATE, Agustín de. Historia del descubrimiento y conquista del Perú. Edición de Franklin Pease G. Y. y Teodoro Hampe Martínez. Lima: Pontificia Universidad del Perú; Fondo editorial, 1995.

ZURIAGA SENENT, Vicent Francesc. La imagen devocional en la Orden de la Merced. Tradición, formación, continuidad y variantes. Valencia: Institució Alfons El Magnànim, 2007. 